\title{
Empirical Models for the Shielding and Reflection of Jet Mixing Noise by a Surface
}

\author{
Cliff Brown * \\ NASA Glenn Research Center, Cleveland, OH, 44135, USA
}

\begin{abstract}
Empirical models for the shielding and reflection of jet mixing noise by a nearby surface are described and the resulting models evaluated. The flow variables are used to nondimensionalize the surface position variables, reducing the variable space and producing models that are linear function of nondimensional surface position and logarithmic in Strouhal frequency. A separate set of coefficients are determined at each observer angle in the dataset and linear interpolation is used to for the intermediate observer angles. The shielding and reflection models are then combined with existing empirical models for the jet mixing and jet-surface interaction noise sources to produce predicted spectra for a jet operating near a surface. These predictions are then evaluated against experimental data.
\end{abstract}

\section{Nomenclature}

$+\quad$ add decibels (logarithmic))

$\bigoplus \quad$ add power (antilogarithmic) observation angle relative to the zenith (azimuthal or roll angle) observation angle relative to the upstream jet axis (polar or yaw angle)

$c_{a} \quad$ speed of sound at ambient conditions

$D_{j} \quad$ nozzle exit diameter

f frequency

$h_{E} \quad$ radial distance from jet lipline to surface (in inches)

$M_{a} \quad$ acoustic Mach number $\left(M_{a}=U_{j} / c_{a}\right)$

$P_{d} \quad$ jet-surface interaction (dipole) noise

$P_{m} \quad$ jet mixing noise

$S P L_{T} \quad$ total sound pressure level

$S t_{D j} \quad$ Strouhal number $\left(S t_{D j}=f D_{j} / U_{j}\right)$

$T_{R} \quad$ total temperature ratio

$U_{j} \quad$ jet exit velocity

$X_{c} \quad$ jet potential core length

$x_{E} \quad$ axial distance from jet exit to surface trailing edge

\section{Background}

GMPIRICAL models are useful in a wide variety of engineering applications where quickly estimating the Eeffect of a design decision is required. Complex systems, consisting of multiple components, often combine the results of many sub-models to provide insight and direction toward a final design decision. The accuracy of these predictions generally depends on the quality and scope of the data used to generate the underlying models and, therefore, the overall result might me improved by expanding or improving the model attached to one component. Aircraft aeroacoustics is one such example of a complex system. In order to predict the noise of an airplane flying at a given altitude, speed, and heading, noise sources from the airframe and the engine components must be combined and the results propagated to the observer location. Each of

*Research Engineer, Acoustics Branch, 21000 Brookpark Rd., AIAA Member. 
these components, however, represent complex noise sources and are, therefore, generally separated into sub-models; an airframe noise prediction might use sub-models for flaps, slats, and landing gear while a prediction of the engine noise uses sub-models for the fan, core, and jet exhaust noise. This approach to noise prediction has functioned reasonably well when applied to the standard tube and wing aircraft that form the bulk of any current fleet. Future aircraft designs often propose tightly integrating the engine and airframe to increase efficiency or reduce noise bringing forth a need for prediction models to capture interactions between the engine and airframe.

Engine exhaust noise prediction tools and methods have been developed to provide the noise spectra generated by a jet plume isolated from the relevant aircraft. This is appropriate for some aircraft where the engine exhaust is not particularly close to the airframe surfaces; isolated jets are also much easier to replicate in a relatively small laboratory than engine-airframe configurations so there is also a vast amount of noise and flow data for available for development and validation. Therefore, a series of tests are being conducted at the NASA Glenn Research Center to provide a noise ${ }^{1-3}$ and flow ${ }^{4}$ database for jets exhausting near surfaces suitable for the development and validation of physics based exhaust noise prediction tools and methods that include the effect of nearby surfaces. Starting with a simple round nozzle near a flat plate (Figure 1), each test phase introduces a new variable increasing the complexity and allowing the prediction methods and models to develop similarly. A core set of jet conditions (Table 1) and parametrically varying surface positions (Table 2) have been acquired during each test phase along with a few points specific to the individual phase. The empirical model, however, is based on the core set to better assess the experimental repeatability and model quality.

Jet-surface interaction (JSI) noise is often the dominant source at low frequencies. ${ }^{1,5,6}$ JSI noise is a source of noise produced as turbulent energy is scattered into acoustic energy as the turbulence passes over the trailing edge of a surface. At higher frequencies, however, the surface is not a strong source of noise but rather acts to shield or reflect the jet mixing noise depending on the position of the surface relative to the jet and observer (Figure 2). This separation between sources and effects is the basis for an empirical jet-surface interaction noise prediction model. ${ }^{7}$ The current model includes two sources, jet mixing noise and jet-surface interaction noise, and two mutually exclusive effects (shielding or reflection) that modify the source spectra. Mathematically, the basic model for the prediction sound pressure level $\left(S P L_{T}\right)$, accounting for the two sources $\left(P_{m}\right.$ and $\left.P_{d}\right)$ and the shielding or reflecting effect $\left(G_{S, R}\right)$, is:

$$
S P L_{T}=P_{m}\left(M_{a}, T_{R}, M_{f}\right)+G_{S, R}\left(M_{a}, T_{R}, x_{E}, h_{E}\right) \oplus P_{d}\left(M_{a}, T_{R}, x_{E}, h_{E}\right)
$$

Note that the shielding or reflecting $\left(G_{S, R}\right)$ modifies the jet mixing source $\left(P_{m}\right)$ rather than the JSI source $\left(P_{d}\right)$ and that sources add as acoustic power $(\oplus)$ while effects add logarithmically (dB values). A model for the JSI source has been developed for a subsonic unheated single stream round jet near a flat surface ${ }^{7}$ and then extended to included hot jet exit conditions. ${ }^{8}$ A source model for an isolated jet, developed by Khavaran and Bridges, ${ }^{9,10}$ can be used to account for the jet mixing noise source. Models for shielding and reflection effects $\left(G_{S, R}\right)$ are now developed. When combined, these modes will predict the complete noise spectra produced by a round jet near a simple surface and measured in the acoustic far-field.

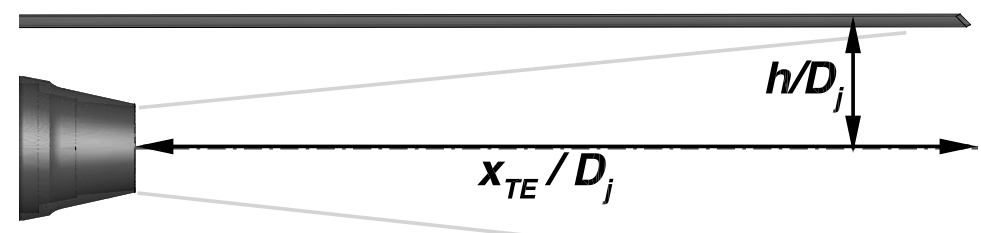

Figure 1. Schematic showing the configuration tested with the nomenclature used to describe the surface and observed locations. 


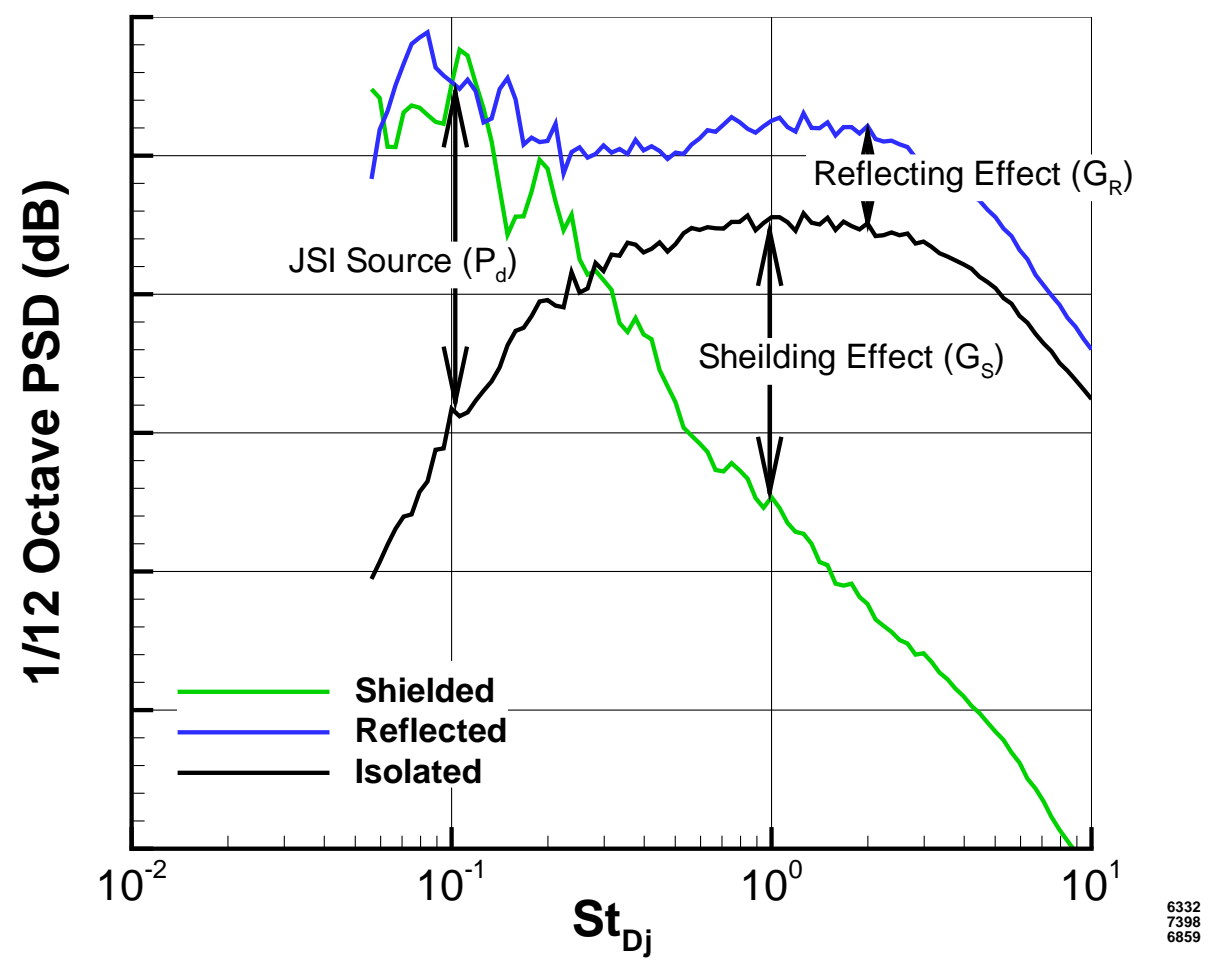

Figure 2. Spectra showing the jet mixing and JSI noise sources with the shielding and reflecting effects.

\begin{tabular}{|c|c|c|c|c|}
\hline Setpoint & $\begin{array}{c}\text { NPR } \\
P_{j} / P_{a}\end{array}$ & $T_{s, j} / T_{a}$ & $\begin{array}{c}M_{a} \\
U_{j} / c_{a}\end{array}$ & $\begin{array}{c}\text { Mass Flow } \\
\mathrm{kg} / \mathrm{s}\end{array}$ \\
\hline 3 & 1.197 & 0.968 & 0.5 & 0.44 \\
\hline 5 & 1.436 & 0.902 & 0.7 & 0.65 \\
\hline 7 & 1.860 & 0.835 & 0.9 & 0.91 \\
\hline 23 & 1.103 & 1.76 & 0.5 & 0.24 \\
\hline 32 & 1.079 & 2.27 & 0.5 & 0.19 \\
\hline 27 & 1.360 & 1.76 & 0.9 & 0.43 \\
\hline 36 & 1.273 & 2.27 & 0.9 & 0.33 \\
\hline 46 & 1.227 & 2.70 & 0.9 & 0.28 \\
\hline
\end{tabular}

Table 1. Unheated subsonic jet exit conditions common to all the jet-surface interaction noise tests. 


\begin{tabular}{|c|c|c|c|c|c|c|c|}
\hline$h$ (inches) & & & $x_{T E}$ (inches) & & & & \\
& 1.3 & 2.7 & 4 & 8 & 12 & 16 & 20 \\
\hline 0.0 & $3,5,7$ & $3,5,7$ & $A L L$ & $A L L$ & $A L L$ & $A L L$ & $A L L$ \\
\hline 0.1 & $3,5,7$ & $3,5,7$ & $3,5,7$ & $3,5,7$ & $3,5,7$ & $3,5,7$ & $3,5,7$ \\
\hline 0.2 & $3,5,7$ & $3,5,7$ & $3,5,7$ & $3,5,7$ & $3,5,7$ & $3,5,7$ & $3,5,7$ \\
\hline 0.3 & $3,5,7$ & $3,5,7$ & $3,5,7$ & $3,5,7$ & $3,5,7$ & $3,5,7$ & $3,5,7$ \\
\hline 0.5 & $3,5,7$ & $3,5,7$ & $A L L$ & $A L L$ & $A L L$ & $A L L$ & $A L L$ \\
\hline 0.7 & $3,5,7$ & $3,5,7$ & $3,5,7$ & $3,5,7$ & $3,5,7$ & $3,5,7$ & $3,5,7$ \\
\hline 1.0 & $3,5,7$ & $3,5,7$ & $A L L$ & $A L L$ & $A L L$ & $A L L$ & $A L L$ \\
\hline 1.4 & $3,5,7$ & $3,5,7$ & $3,5,7$ & $3,5,7$ & $3,5,7$ & $3,5,7$ & $3,5,7$ \\
\hline 1.5 & & & $A L L$ & $A L L$ & $A L L$ & $A L L$ & $A L L$ \\
\hline 1.9 & $3,5,7$ & $3,5,7$ & $3,5,7$ & $3,5,7$ & $3,5,7$ & $3,5,7$ & $3,5,7$ \\
\hline 2.0 & & & $A L L$ & $A L L$ & $A L L$ & $A L L$ & $A L L$ \\
\hline 2.5 & $3,5,7$ & $3,5,7$ & $A L L$ & $A L L$ & $A L L$ & $A L L$ & $A L L$ \\
\hline 3.0 & & & $A L L$ & $A L L$ & $A L L$ & $A L L$ & $A L L$ \\
\hline 3.2 & $3,5,7$ & $3,5,7$ & $3,5,7$ & $3,5,7$ & $3,5,7$ & $3,5,7$ & $3,5,7$ \\
\hline 4.0 & $3,5,7$ & $3,5,7$ & $A L L$ & $A L L$ & $A L L$ & $A L L$ & $A L L$ \\
\hline 5.0 & $3,5,7$ & $3,5,7$ & $A L L$ & $A L L$ & $A L L$ & $A L L$ & $A L L$ \\
\hline
\end{tabular}

Table 2. Surface lengths $\left(x_{T E} / D_{j}\right)$ and radial distances $(h)$ used to generate the empirical model. The numbers in each box represent the setpoint tested at that surface location ( $A L L$ indicates that all setpoints in Table 1).

\section{Separation of Sources and Effects}

The exhaust noise model presented above (Equation 1 allows the flexibility to consider noise source and effects independently. While this is considered a strength of the model because it allows independent modeling of different physical sources (e.g. the dipolar JSI source versus the quadrupolar jet mixing source) and allows the model to incorporate additional sources and effects as new data are acquired (e.g. nozzle aspect ratio, effect of flight, separate flow nozzles, etc.), it requires that the each source and effect be separated from the measured spectra. The first step is to separate the noise sources. In this case, theoretical ${ }^{11-13}$ and experimental ${ }^{3,5,6}$ research has shown that the JSI noise source is localized and dipolar in nature and, therefore, coherent across a range of observer angles where the jet mixing noise is spatially incoherent. This property allows the JSI source spectrum to be separated from the measured far-field spectrum $\left(S P L_{T}\right)^{7}$ leaving the jet mixing source and shielding / reflection effect. If the jet mixing noise $\left(P_{m}\right)$ is measured independently (by removing the surface) then a simple rearrangement of the empirical model equation then allows the shielding or reflecting effect to be determined as:

$$
G_{S, R}\left(M_{a}, T_{R}, x_{E}, h_{E}\right)=\left(S P L_{T} \ominus P_{d}\left(M_{a}, T_{R}, x_{E}, h_{E}\right)\right)-P_{m}\left(M_{a}, T_{R}, M_{f}\right)
$$

where $\ominus$ indicates subtraction of acoustic power and - indicates subtraction on a logarithmic (dB) basis. Figures 3 and 4 show an example of the spectral separation for the shielded and reflected surface configurations. Note that the separation procedure used data in 1/12-octave spectral density to provide some smoothing while retaining a sufficient number of spectral points to create the model.

Figure 3 shows an example of separating the shielding effect from the measured spectra and Figure 4 shows a representative separation of the reflecting effect (at the same surface length, standoff, and jet conditions).

The source separation using the coherence of real signals is not perfect; the large variations when $S t_{D j}<1$ in Figures 3 and 4 are the result of these variations in coherence and the direct spectral subtraction used to separate the noise sources and effects. As a result, a methodology for setting the minimum frequency above which the data are valid is needed before attempting to model the effect.

In the shielded configuration, if refraction is neglected, each mixing noise source is either covered by the surface (shielded) or allowed to propagate to the observer (unshielded). If line of sight propagation of sound 
is assumed, then a line between the observer location and the surface trailing edge will point to an axial location, $x_{s}$, on the jet centerline that divides the shielded from the unshielded sources (Figure 5(a)). This point will be a function of the observer location $\left(x_{o}, y_{o}\right)$, the surface length $\left(x_{E}\right)$, surface standoff distance $\left(h_{E}\right)$, and nozzle diameter $\left(D_{j}\right)$ so that:

$$
x_{s}=x_{E}-\frac{\left(x_{o}-x_{E}\right)}{\left(y_{o}-h_{E}-D_{j} / 2\right)}\left(h_{E}+D_{j} / 2\right)
$$

Note the additional term $D_{j} / 2$ is included because $h_{E}$ is measured from the nozzle lipline and $x_{s}$ is defined on the jet centerline. The jet mixing noise sources located upstream of $x_{s}$ will be shielded while the sources downstream of $x_{s}$ will not.

In the reflected configuration (Figure 5(b)), again neglecting refraction, it is assumed that the angle of incidence to the surface is equal to the angle for reflection and, therefore, only mixing noise sources upstream of the point $x_{s}$ will reflect to the observer. The point dividing the reflecting sources from the non-reflecting sources $\left(x_{s}\right)$ for an observer at $\left(x_{o}, y_{o}\right)$ is:

$$
x_{s}=x_{E}-\frac{\left(x_{o}-x_{E}\right)}{\left(y_{o}+h_{E}+D_{j} / 2\right)}\left(h_{E}+D_{j} / 2\right)
$$

The jet mixing noise sources located upstream of $x_{s}$ will be reflected while the sources downstream of $x_{s}$ will not. However, like the shielded configuration, solving this geometry problem provides a location dividing the relevant sources but does not provide frequency information that might be used to form a model acoustic spectra.

Jet mixing noise is represented by a distribution of sources. Phased array data shows that the higher frequency components where the higher frequencies originate near the nozzle while the lower frequency sources are located farther downstream. Such source location data can be used to relate a location on the jet centerline to the dominate frequency at that point. Figure 6 shows phased array data acquired by Podboy for isolated (no surface) jets operating at four of the setpoints listed in Table 1. Note that the source distribution collapses across this range of jet operating conditions when nondimensionalized by the jet potential core length. This allows a linear equation to describe the source location as:

$$
\frac{x_{s}}{X_{c}}=\operatorname{maximum}\left(-0.8014 \log _{10}\left(S t_{D j}\right)+0.6859,0\right)
$$

where $X_{c}$ is the jet potential core length and a maximum function is used to prevent the source location from moving inside the nozzle (which is a consequence of fitting a linear function of $\log _{10}\left(S t_{D j}\right)$ rather than using an standard exponential function). Finally, combining Equation 3 or 4 with Equation 5 the maximum frequency for shielding or reflection can be estimated.

\section{Shielding Effect Model}

Jet mixing noise is characterized as a series of noise sources distributed throughout the jet plume. Phased array source location data shows that these sources are distributed logrithmically along the jet axis according to frequency (Figure 6). An analysis of the shielding effect, separated from the measure spectra, shows a similar logarithmic behavior when plotted as a function of Strouhal frequency (Figure 3(b)). Assuming a logarithmic spectral model for the shielding effect, given a surface position, jet velocity, and jet temperature, leads to the model equation:

$$
G_{S}\left(S t_{D j}\right)=C_{1, S} * \log _{10}\left(S t_{D j}\right)+C_{2, S}
$$

where $C_{1, S}$ and $C_{2, S}$ are coefficients determined by fit to the spectral data. This model is extended to other jet conditions and surface positions by first finding the coefficient values $C_{1, S}$ and $C_{2, S}$ for each measured spectra and then creating an equation model for each coefficient as a function of jet Mach number $\left(M_{a}\right)$, jet temperature ratio $\left(T_{R}\right)$, surface length $\left(x_{E}\right)$ and surface standoff distance $\left(h_{E}\right)$. The resulting model can then be used by working the process in reverse; the relevant flow and surface variables are fed into the coefficient models to get values for $C_{1, S}$ and $C_{2, S}$ which are then used in Equation 6 to estimate the shielding effect.

The challenge of creating a viable model for the shielding effect is the number of independent variables; a single stream jet flow is described by a Mach number and a temperature ratio, the surface position is given by 


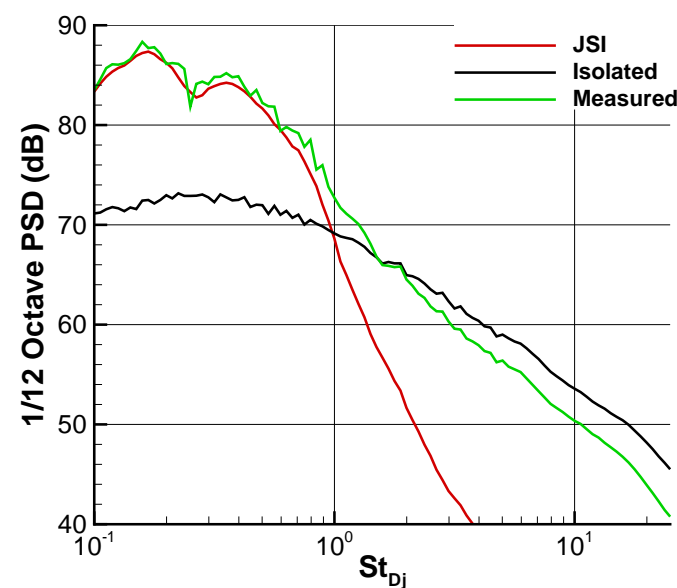

(a) Source Spectra

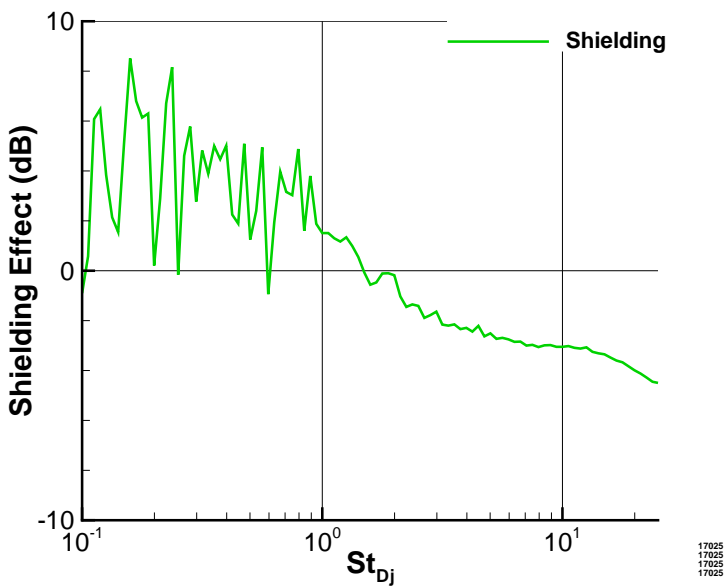

(b) Shielding Effect

Figure 3. Spectra measured by the shielded observer at setpoint 3 with a surface at $x_{E}=8$ " and $h_{E}=0.5$ " with the JSI noise, extracted using the coherence method, and the independently measured jet mixing noise (3(a)). Applying the effect separation technique (Equation 2) to these spectra gives the shielding effect $(3(\mathrm{~b}))$.

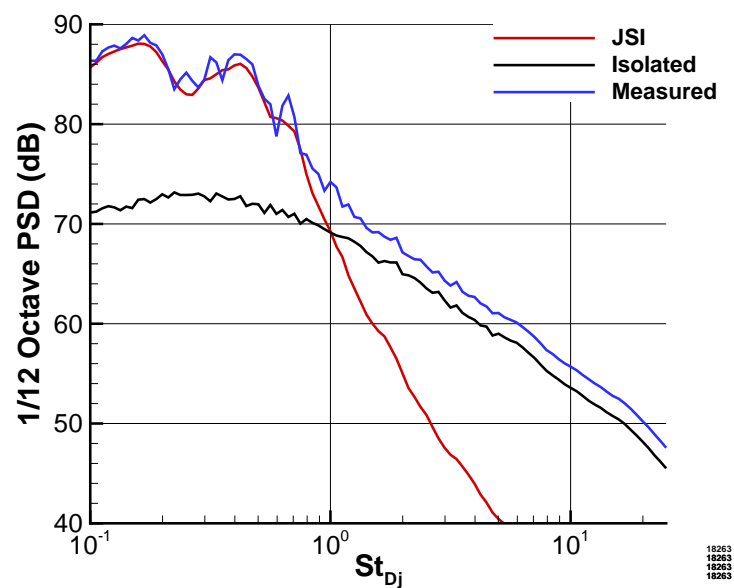

(a) Source Spectra

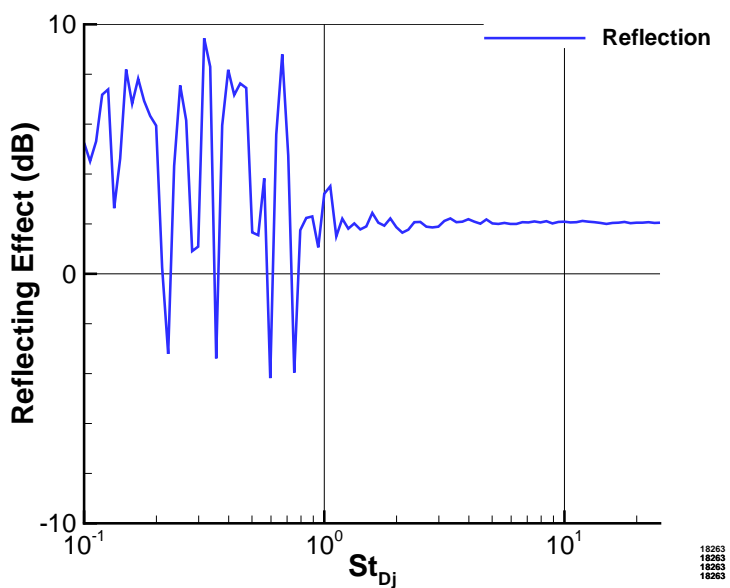

(b) Reflection Effect

Figure 4. Spectra measured by the reflected observer at setpoint 3 with a surface at $x_{E}=8 "$ and $h_{E}=0.5$ " with the JSI noise, extracted using the coherence method, and the independently measured jet mixing noise (4(a)). Applying the effect separation technique (Equation 2) to these spectra gives the reflection effect $(4(b))$. 


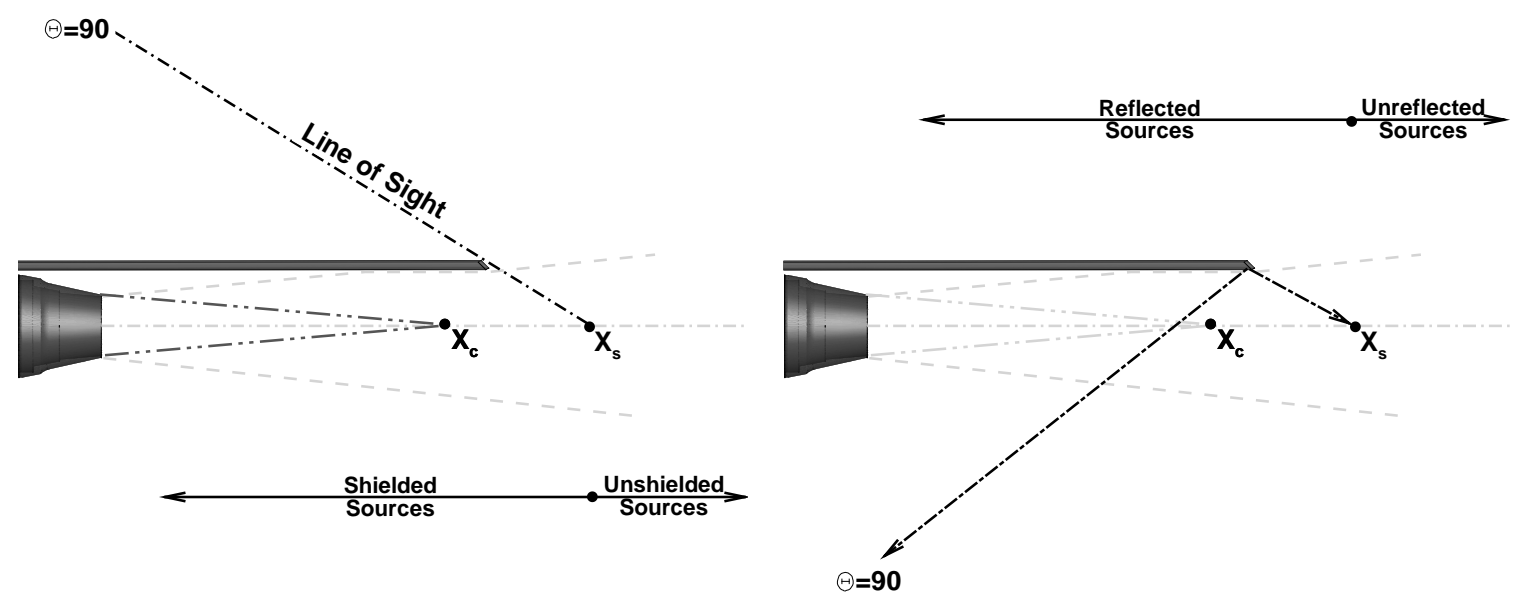
(a) Source Spectra
(b) Reflection Effect

Figure 5. Setup for determining the limiting source location for the shielding and reflection surface configurations.

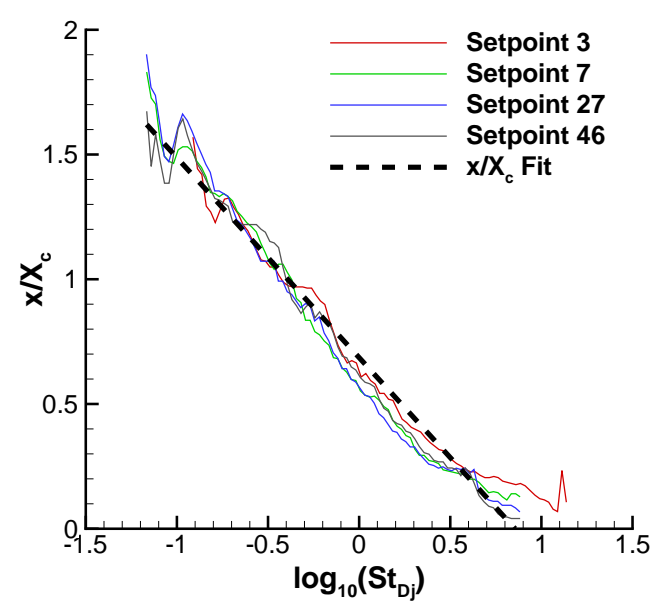

Figure 6. Phased array source location data ${ }^{2}$ compared to the results from Equation 5. 
the surface length and standoff distance, and the observer location requires another three variables, $\left(x_{o}, y_{o}, z_{o}\right)$ or $(R, \theta, \phi)$, for definition. Thus, including spectral frequency, the shielding effect model is operating in an 8-dimensional variable space. Fortunately, the number of independent variables can be reduced in three ways: creating multiple models and interpolating between the results, assuming a functional form based on observation, or variable nondimensionalization. The shielding effect model uses all three of these techniques. First, a set of coefficients is determined for each observer location in the data set so that a low-order interpolation can be used on the results (this is simplified by the semi-infinite surface leaving only one azimuthal angle). Next, a function form, given by Equation 6, is assumed for the spectral shape. Finally, nondimensionalization is used to reduce the jet flow and surface position from four independent variables to only two.

Only a subset of the jet mixing noise sources, based on the relative position of the source, surface, and observer, are surface subject to the shielding effect. The surface position and the observer location can be measured or provided from a conceptual design. The source locations, however, are vary with the jet velocity and temperature which exist in the shielding model to represent the distribution of source along the jet centerline. Phased array source localization data has shown that the source distribution collapses across a range of heated and unheated single stream jets if the axial variable is nondimensionalized by the jet potential core length (e.g. Figure 6). Furthermore, since the relative location of the source to the surface is of primary importance, jet velocity and temperature can be eliminated as independent variables if the surface length is nondimensionalized by the jet potential core length. Note that these variables are still needed to define a configuration but are now used only to compute the potential core length.

The surface standoff distance also changes which sources are shielded; as the surface moves away from the jet, more sources will be shielded from an upstream observer while being revealed to the downstream observer. This process depends not only on the source distribution and the surface standoff distance but also the surface length; a small change in standoff distance is more significant when the surface is short than when it is long. Thus, it is really the angle between the source and the surface that determines if that source is shielded. The effect of a change in surface standoff is then best represented when nondimensionalized by the surface length.

The basic model equation for the shielding effect as a function of Strouhal frequency has two coefficients that are functions of the nondimensional surface position $\left(x_{E} / X_{c}\right.$ and $\left.h_{E} / x_{E}\right)$ and must be determined by fitting a model equation to the available data. The model equation selected for these coefficients is linear in non-dimensional surface length and surface standoff distance with a constant term written mathematically as:

$$
\begin{aligned}
& C_{1, S, \theta}\left(x_{E} / X_{c}, h_{E} / x_{E}\right)=K_{1, S, \theta}+K_{2, S, \theta}\left(x_{E} / X_{c}\right)+K_{3, S, \theta}\left(h_{E} / x_{E}\right) \\
& C_{2, S, \theta}\left(x_{E} / X_{c}, h_{E} / x_{E}\right)=K_{4, S, \theta}+K_{5, S, \theta}\left(x_{E} / X_{c}\right)+K_{6, S, \theta}\left(h_{E} / x_{E}\right)
\end{aligned}
$$

where the coefficients $K_{1-3, S, \theta}$ and $K_{4-6, S, \theta}$ are determined by a singular value decomposition fit to the $C_{1, S}$ and $C_{2, S}$ data respectively (Appendix A). Two notes on the model implementation should be included here: (1) positive values, which are non-physical for the shielding effect, will occur if the model is extended across the entire frequency range and (2) the linear $\left(C_{1, S, \theta}\right)$ term, can be close to zero, particularly at downstream observer angles where the shielding effect is relatively weak, so that the model extends the shielding effect over the entire range of frequencies rather than only the shielded subset. The first issue is addressed by setting a maximum value of zero on the shielding effect. The second problem is addressed by adding a suppression function $\left(S\left(S t_{D j}\right)\right)$ that activates at the minimum frequency $\left(f_{\min }\right)$ used for separating the shielding effect from the measured spectra (Equation 5) to limit the frequency range of the model. The suppression function in the current model is the logistic function:

$$
S\left(S t_{D j}\right)=\frac{1}{1+e^{-\alpha\left(S t_{D j}-f_{o}\right)}}
$$

where $\alpha$ is a constant selected to control the rate of decrease ( $\alpha=8$ in the current implementation) and $f_{o}$ is the inflection point computed so that the $S\left(f_{\min }\right)=0.99$ using the equation:

$$
f_{o}=\frac{1}{\alpha} \ln \left(\frac{1}{0.99}-1\right)+f_{\min }
$$


The uncertainty of the shielding effect model is difficult to determine quantitatively because of variations in the underlying effect data. Figure 7 shows a comparison between the shielding effect separated from the measured data and the modeled effect at a surface and jet flow near the center of the variable space (setpoint $\left.5, x_{E} / X_{c}=1.1\right), h_{E} / x_{E}=0.042$ ). At $\theta=90^{\circ}$ the model has a slope that reasonably captures the shielding effect for $S t_{D j}>1$. Below this frequency there is residual noise from the effect separation related to the combination of the jet mixing noise source and the the jet-surface interaction noise source. Note how limiting the maximum value to zero creates a natural end point for the shielding effect when the linear term is significant. The separated effect at $\theta=150^{\circ}$ has an entirely different character. First, the amplitude at low frequency is negative which appears to be caused by too much energy being categorized in the jet-surface interaction source. However, this result implies a shielding effect at these frequencies which the assumptions underlying the shielding effect model do not support (e.g. based on line-of-sight these sources are exposed). The minimum frequency for this configuration at $\theta=150^{\circ}$ is predicted at $f_{\text {min }} \approx 0.45$ with a linearly decreasing trend (increased shielding) at higher frequencies. Initially this trend is observed but then there is a decrease in shielding for $S t_{D j}>3.5$ that is not expected. The result is that the linear fit across this frequency range picks up the peak points on either end and misses the decrease in the middle. Because of this, the model, In general, performs better at upstream and broadside observer angles and has more uncertainty for downstream observer angles. Table 3 contains an estimate for the root-mean-square deviation (RMSD) and normalized root-mean-square deviation (NRMS) based on overall sound pressure level computed starting at $f_{\min }$ for each setpoint and surface position in the experimental data base.

The shielding effect is one part of the exhaust noise system described by Equation 1. Figure 8 shows the results of combining the shielding effect with a jet mixing noise model ${ }^{9,10}$ and an empirical model for the jet-surface interaction noise source. ${ }^{8}$ These plots, show two trends in the overall prediction: (1) the parabolic shape at high frequencies and downstream angles (as in Figure 7(b)) appears as an area of increased difference at all 3 setpoints shown and (2) the difference between the model and measured data increase at higher frequencies as the jet condition increases. This second point is actually related to another point; the model has more uncertainty for shorter surfaces (a longer jet potential core with a higher velocity setpoint is equivalent to a shorter surface in the model). The model should smoothly trend to the isolated jet (i.e. zero shielding effect) as the surface becomes very short but, because the equation used are all first-order, the model does not smoothly go to zero with surface length. Rather, it is biased by the longer surfaces that do exhibit more of a linear variation resulting in the observer issues. Therefore, a modification to model introducing higher order terms, at least in surface length, should be considered if these shorter surfaces are of primary interest.

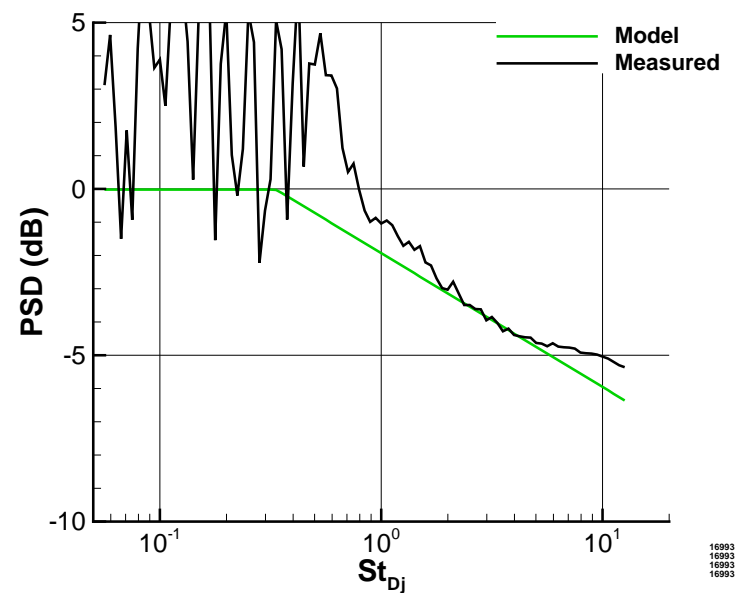

(a) $\theta=90^{\circ}$

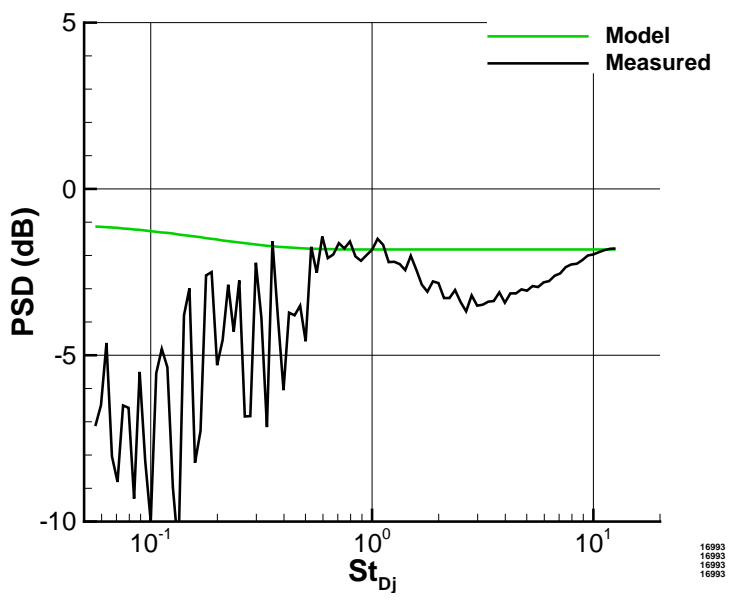

(b) $\theta=150^{\circ}$

Figure 7. Measured and modeled shielding effect for a jet at setpoint $\mathbf{5}$ with a surface at $x_{E}=12$ ", $h_{E}=0.5 "$. 


\begin{tabular}{|c|c|c|c|c|}
\hline & $\theta=60^{\circ}$ & $\theta=90^{\circ}$ & $\theta=120^{\circ}$ & $\theta=150^{\circ}$ \\
\hline RMSD & 1.853 & 1.671 & 1.956 & 3.212 \\
\hline NRMSD & 0.162 & 0.206 & 0.308 & 0.741 \\
\hline
\end{tabular}

Table 3. Root-mean-square deviation (RMSD) and normalized root-mean-square deviation (NRMS) for the shielding effect model at observer angles $\theta=60^{\circ}, \theta=90^{\circ} . \theta=120^{\circ}$, and $\theta=150^{\circ}$. RMSD was computed on the values of the shielding effect (e.g. 7) integrated over $S t_{D j}>f_{\min }$ for every surface position and setpoint in the experimental database.

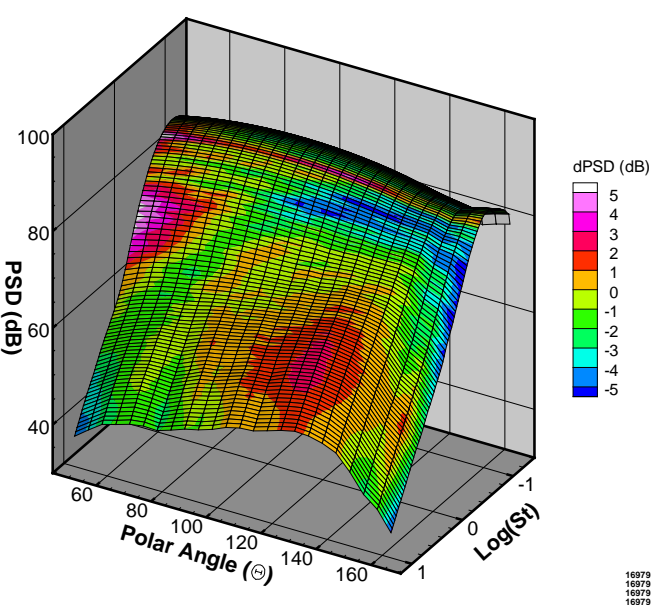

(a) Setpoint 3

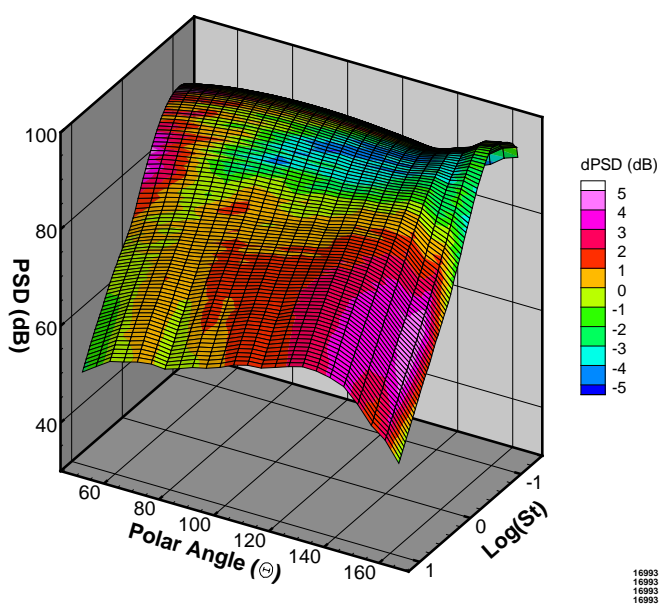

(b) Setpoint 5

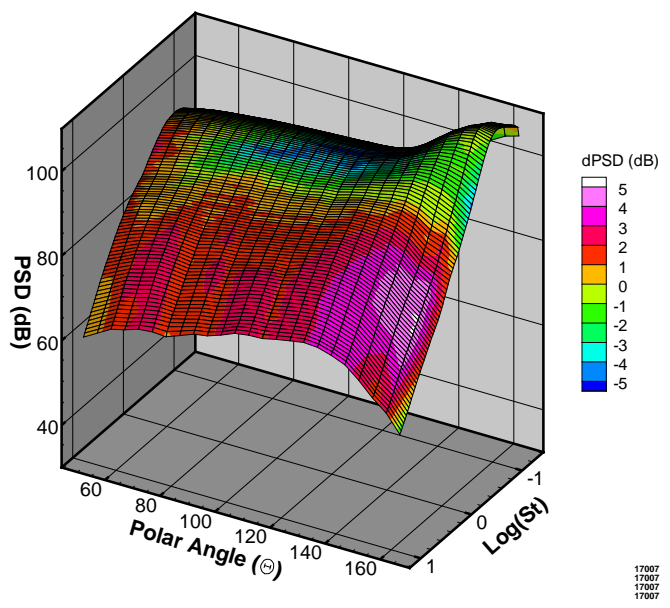

(c) Setpoint 7

Figure 8. Spectra predicted for the shielded observer with a surface at $x_{E}=12$ " and $h_{E}=0.5$ " and setpoints $3, \mathbf{5}, \mathbf{7}$. Note that the color represents $\triangle P S D=P S D_{\text {model }}-P S D_{\text {measured }}$. 


\section{Reflection Effect Model}

The underlying physics of shielding and reflection effects are similar; generally a source that is shielded to one observer will be reflected to another observer. The reflection model, therefore, is very similar to the shielding model with one important difference; because jet noise is produced by a distribution of incoherent sources the theoretical maximum reflection is $3 \mathrm{~dB}$ where the full energy of a source can be shielded. This physical difference results in a compressed range of values and, therefore, a very weak linear term in model equation. Thus the linear term is excluded from the refection model so that the reflection effect $\left(G_{R}\right)$ is given by the equation:

$$
G_{R}\left(S t_{D j}\right)=S\left(S t_{D j}\right) * C_{2, R}
$$

where is the suppression function $S\left(S t_{D j}\right)$, as given in Equation 9, is used to limit the frequency range and $C_{2, R}$ is determined by a singular value decomposition fit to the available data. The same logistic function used by the shielding effect model (Equation 9) is used in the reflecting model. Furthermore, because the reflecting model relies on the same source distributing as the shielding model, the form of the coefficient equation $C_{2, R}$ is the same given by:

$$
C_{2, R, \theta}\left(x_{E} / X_{c}, h_{E} / x_{E}\right)=K_{1, R, \theta}+K_{2, R, \theta}\left(x_{E} / X_{c}\right)+K_{3, R, \theta}\left(h_{E} / x_{E}\right)
$$

where the surface length is nondimensionalized by jet potential core length and the surface standoff distance is nondimensionalized by the surface length. The determined values for the three coefficients $K_{1, R, \theta}, K_{2, R, \theta}$, and $K_{3, R, \theta}$ are provided in Appendix B.

Figure 9 shows the reflection effect extracted from the measured spectra and modeled using equations 11 and 12. At $\theta=90^{\circ}$, the experimental data shows a reflection effect with a parabolic shape at $S t_{D j}>1$ that is not captured by the constant value model. The model does, however, provide a reasonable estimate of the effect at either end of the range. At $\theta=150^{\circ}$, the minimum reflected frequency, based on the line-of-sight assumption (Equation 4), is above the maximum frequency shown so the model never returns a non-zero value. The extracted data shows an increase of less that $1 \mathrm{~dB}$ across the range $1<S t_{D j}<10$ which could be attributed to reflection. This may be a limitation of using a simple geometric estimation of the frequency range or this increase could be caused by another factor such as refraction off the surface trailing edge. Table 4 shows the RMSD and NRMSD for observer angles $\theta=60^{\circ}, \theta=90^{\circ}, \theta=120^{\circ}$, and $\theta=150^{\circ}$ based on the overall sound pressure level computed over frequencies where reflection effect is present. In contrast to the shielding effect model, the reflection model performs better based on RMSD at downstream observer angles than at upstream angles although it is fairly consistent across all angles on a normalized basis. However, as noted in the analysis of the shielding effect model, there is considerable noise in these data so the RMSD should be used as a guide to model performance rather than an absolute statement of uncertainty.

Finally, the reflection model has been integrated into an overall empirical prediction of exhaust noise for a jet near a surface based on Equation 1. Figure 10 shows an example of the results for predictions for a $x_{E}=12$ " surface $h_{E}=0.5$ " from the jet. The largest region of difference is at downstream angles $\left(\theta>100^{\circ}\right)$ at all three setpoints. Interestingly, the greatest difference occur at setpoint 5 , in the middle of the range, indicating clearly some nonlinearities that are not captured by the model. This implies that the model could benefit from introducing higher order terms of surface length into Equation 12 (which is the term closely related to jet condition). The current model, however simple, is probably best suited to system level studies designed to screen a wide range of configurations for future study. In this application, this model benefits from a minimum number of coefficients and overall simplicity.

\begin{tabular}{|c|c|c|c|c|}
\hline & $\theta=60^{\circ}$ & $\theta=90^{\circ}$ & $\theta=120^{\circ}$ & $\theta=150^{\circ}$ \\
\hline RMSD & 2.830 & 1.419 & 0.843 & 0.805 \\
\hline NRMSD & 0.173 & 0.288 & 0.221 & 0.207 \\
\hline
\end{tabular}

Table 4. Root-mean-square deviation (RMSD) and normalized root-mean-square deviation (NRMS) for the reflected effect model at observer angles $\theta=60^{\circ}, \theta=90^{\circ} \cdot \theta=120^{\circ}$, and $\theta=150^{\circ}$. RMSD was computed on the values of the refelcted effect (e.g. Figure 9) integrated over $S t_{D j}>f_{m i n}$ for every surface position and setpoint in the experimental database. 


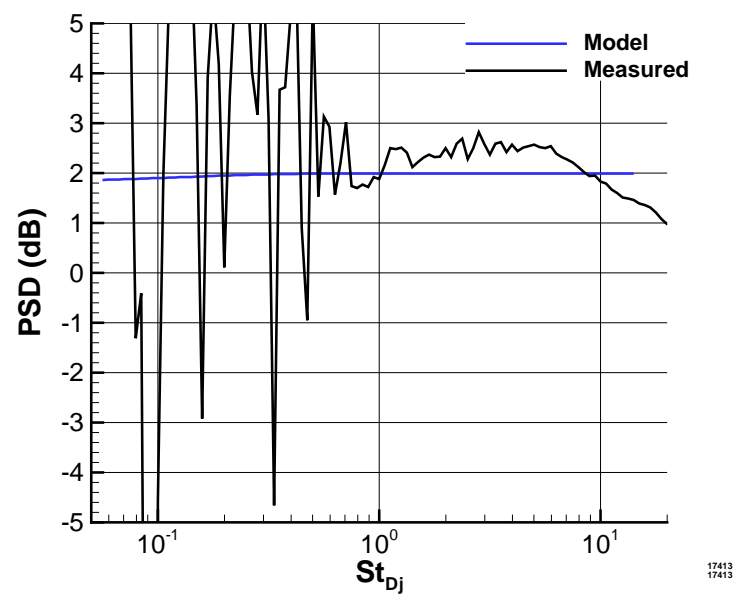

(a) $\theta=90^{\circ}$

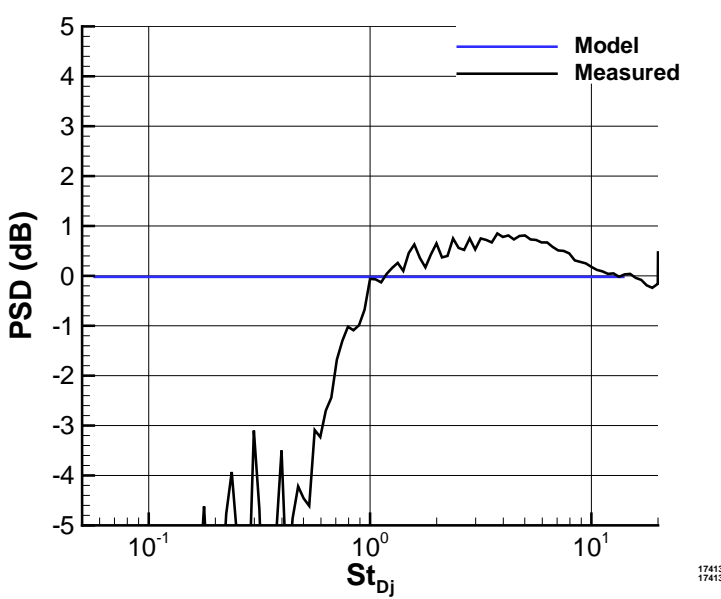

(b) $\theta=150^{\circ}$

Figure 9. Measured and modeled reflection effect for a jet at setpoint $\mathbf{5}$ with a surface at $x_{E}=12$ ", $h_{E}=0.5 "$.

\section{Conclusions}

Empirical models have been developed for the shielding and reflection of jet mixing noise by a surface. These models, combined with existing models for jet mixing noise and jet-surface interaction noise, allow for prediction of the complete spectra produced by a simple surface is near a simple, single flow, subsonic round jet. Jet velocity and temperature are accounted for by nondimensionalizing the surface length by the jet potential core length and surface standoff distance by the surface length to create a stand-in for the minimum shielding or reflection angle. Both the shielding and reflection models are linear nondimensional surface length, which may limit model performance in some configurations, and nondimensional surface standoff distance. These models are suitable for use in system level noise prediction models to design the next generation of aircraft.

\section{Acknowledgements}

This work was supported by the NASA Fundamental Aeronautics Program, Advanced Air Transportation Technologies and Commercial Supersonic Transport Projects. The author would like to thank Gary Podboy for providing the phased array source localization data and James Bridges for helping to acquire the far-field noise data as well as reviewing this manuscript.

\section{References}

1, Brown, C., "Jet-Surface Interaction Test: Far-Field Noise Results", ASME GT2012-69639, 2012.

${ }^{2}$ Podboy, G., "Jet-Surface Interaction Test: Phased Array Noise Source Localization Results", ASME GT2012-69801, 2012.

${ }^{3}$ Brown, C.A. , "Jet-Surface Interaction Test: Far-Field Noise Results", J. Eng. Gas Turbines Power, 135(7), Jun. 2013.

${ }^{4}$ Brown, C., "Jet-Surface Interaction Test: Far-Field Noise Results", to be presented at the 2014 AIAA Aviation Forum.

${ }^{5} \mathrm{Head}, \mathrm{R} . W$. and Fisher, M.J., "Jet/Surface Interaction Noise: Analysis Of Farfield Low Frequency Augmentation of Jet Noise Due To The Presence Of A Solid Surface", AIAA 1976-502, 1976.

${ }^{6}$ Lawrence, J.L.T., Azarpeyvand, M., and Self, R.H., "Interaction between a Flat Plate and a Circular Jet", AIAA 20112745,2011

${ }^{7}$ Brown, C., "Developing an Empirical Model for Jet-Surface Interaction Noise", AIAA 2014-0878, 2014.

${ }^{8}$ Brown, C., "An Empirical Jet-Surface Interaction Noise Model with Temperature and Nozzle Aspect Ratio Effects", to be presented at the 2015 AIAA SciTech Conference.

${ }^{9}$ Khavaran, A. and Bridges, J., "Development of jet noise power spectral laws using SHJAR data", AIAA 209-3378, 2009.

${ }^{10}$ Khavaran, A. and Bridges, J., "SHJAR jet noise data and power spectral laws", NASA/TM 2009-215608, 2009. 


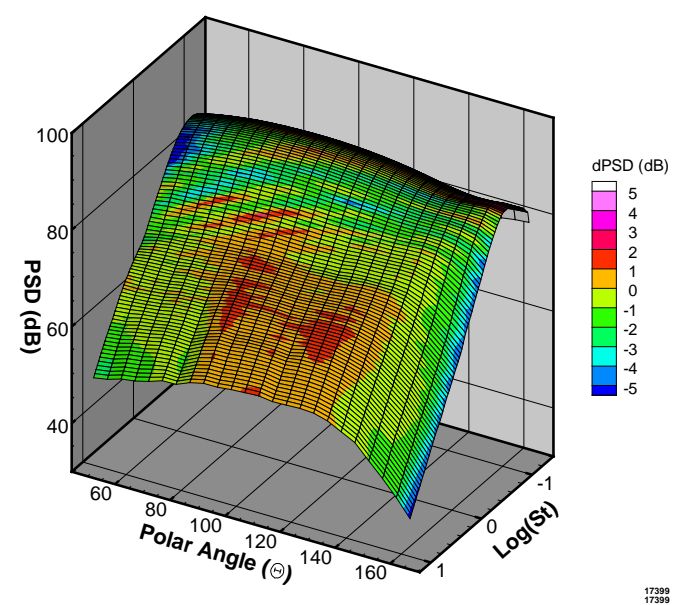

(a) Setpoint 3

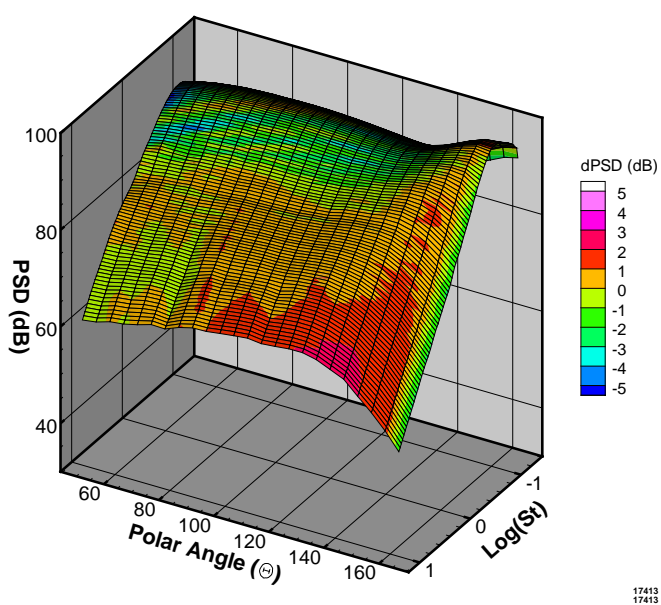

(b) Setpoint 5

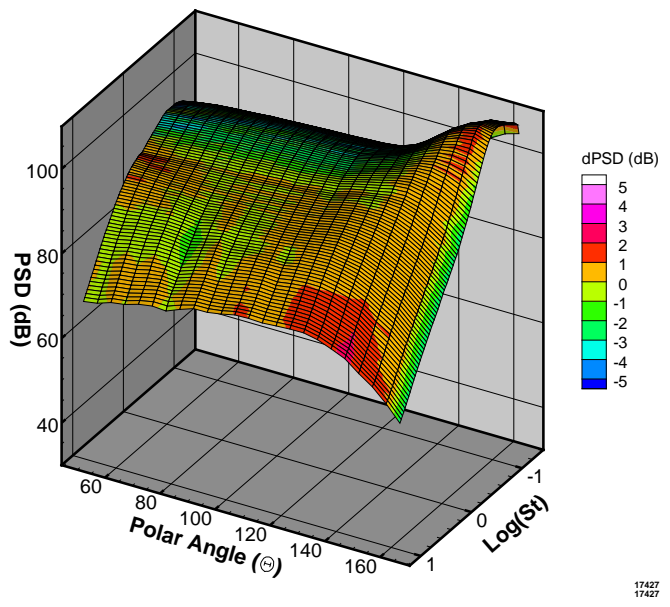

(c) Setpoint 7

Figure 10. Spectra predicted for the reflection observer with a surface at $x_{E}=12$ " and $h_{E}=0.5$ " and setpoints $3, \mathbf{5}, \mathbf{7}$. Note that the color represents $\triangle P S D=P S D_{\text {model }}-P S D_{\text {measured }}$. 

1955.

${ }^{11}$ Curle, N., "The influence of solid boundaries upon aerodynamic sound", Proc. Roy. Soc. of London, 231A, pp. 505-514,

${ }^{12}$ Ffowcs-Williams, J.E. and Hall, L.H., "Aerodynamic sound generation by turbulent flow in the vicinity of a scattering half plane", J. Fluid Mech., 40(4), pp. 657-670, 1970.

${ }^{13}$ Crighton, D.G. and Leppington, F.G., "Scattering of aerodynamic noise by a semi-infinite compliant plate", J. Fluid Mech., 43(4), pp. 721-736, 1970.

${ }^{14}$ Witze, P., "Centerline velocity decay of compressible free jets", AIAA J. 12(4), pp 417-418, 1974.

\section{Appendix A. Coefficients for Shielding Effect Model}

The basic shielding effect model is given by the equation:

$$
G_{S}\left(S t_{D j}\right)=C_{1, S} * \log _{10}\left(S t_{D j}\right)+C_{2, S}
$$

where $C_{1, S}$ and $C_{2, S}$ are determined by a fit to the available data at each observer angle. In this fit $C_{1, S}$ and $C_{2, S}$ have the functional form:

$$
\begin{aligned}
& C_{1, S, \theta}\left(x_{E} / X_{c}, h_{E} / x_{E}\right)=K_{1, S, \theta}+K_{2, S, \theta}\left(x_{E} / X_{c}\right)+K_{3, S, \theta}\left(h_{E} / x_{E}\right) \\
& C_{2, S, \theta}\left(x_{E} / X_{c}, h_{E} / x_{E}\right)=K_{4, S, \theta}+K_{5, S, \theta}\left(x_{E} / X_{c}\right)+K_{6, S, \theta}\left(h_{E} / x_{E}\right)
\end{aligned}
$$

The constants $K_{1-3, S, \theta}$ for $C_{1, S, \theta}$ and the constants $K_{4-6, S, \theta}$ for $C_{2, S, \theta}$ are provided in Table 5 .

\begin{tabular}{|c|c|c|c|c|c|c|}
\hline$\theta$ & $K_{1, S, \theta}$ & $K_{2, S, \theta}$ & $K_{3, S, \theta}$ & $K_{4, S, \theta}$ & $K_{5, S, \theta}$ & $K_{6, S, \theta}$ \\
\hline 50 & -0.2211 & -3.7826 & -0.2474 & 0.6517 & -5.9566 & -1.1774 \\
\hline 55 & -0.1866 & -3.4739 & -0.1259 & 1.6067 & -5.796 & -1.0629 \\
\hline 60 & -0.3795 & -3.0797 & -0.0096 & 2.0904 & -5.4773 & -0.9502 \\
\hline 65 & -0.4748 & -3.0364 & 0.0866 & 2.3012 & -5.1036 & -0.8407 \\
\hline 70 & -0.5845 & -3.0888 & 0.167 & 2.4712 & -4.7913 & -0.7671 \\
\hline 75 & -0.8087 & -3.193 & 0.2078 & 2.5903 & -4.5956 & -0.6998 \\
\hline 80 & -0.678 & -3.2788 & 0.2272 & 2.546 & -4.3263 & -0.6208 \\
\hline 85 & -0.6605 & -3.1594 & 0.2355 & 2.4131 & -4.0524 & -0.5419 \\
\hline 90 & -0.3759 & -3.3265 & 0.3384 & 2.1933 & -3.7127 & -0.5595 \\
\hline 95 & -0.056 & -3.3038 & 0.0842 & 1.8474 & -3.4057 & -0.2258 \\
\hline 100 & 0.0439 & -3.2758 & 0.0265 & 1.7998 & -3.289 & -0.2007 \\
\hline 105 & 0.427 & -3.0599 & -0.7651 & 1.5882 & -3.168 & 0.3822 \\
\hline 110 & 0.1906 & -2.7663 & -0.6983 & 1.8359 & -3.2541 & -0.0311 \\
\hline 115 & 0.1017 & -2.5859 & -0.4095 & 1.9069 & -3.1062 & -0.5439 \\
\hline 120 & 0.4684 & -2.372 & -1.1474 & 1.4908 & -2.8588 & -0.1286 \\
\hline 125 & 0.3645 & -1.8174 & -1.543 & 1.5062 & -2.8973 & -0.3800 \\
\hline 130 & 0.5858 & -1.6515 & -1.7425 & 1.1487 & -2.4776 & -0.7817 \\
\hline 135 & 0.7324 & -1.2731 & -1.5989 & 0.8484 & -2.2497 & -1.1468 \\
\hline 140 & 0.6219 & -0.596 & -2.1725 & 0.7519 & -2.1687 & -1.3541 \\
\hline 145 & 0.3973 & -0.0769 & -2.4692 & 0.9082 & -2.2538 & -1.7521 \\
\hline 150 & 0.157 & 0.3247 & -2.158 & 0.7406 & -2.2163 & -2.6198 \\
\hline 155 & 0.0845 & 0.0029 & 1.9909 & -0.3135 & -2.3131 & 1.8876 \\
\hline 160 & -0.3026 & 0.9065 & -5.2792 & -0.6623 & -1.2156 & -1.0916 \\
\hline 165 & -0.1918 & 0.8343 & -4.3896 & -0.7527 & -1.5226 & -1.039 \\
\hline
\end{tabular}

Table 5. Coefficients $K_{1-3, S, \theta}$ used by the shielding effect model. 


\section{Appendix B. Coefficients for Shielding Effect Model}

The basic reflection effect model is given by the equation:

$$
G_{R}\left(S t_{D j}\right)=S\left(S t_{D j}\right) * C_{2, R}
$$

where $C_{2, R}$ determined by a fit to the available data at each observer. In this fit, $C_{2, R}$ has the functional form:

$$
C_{2, R, \theta}\left(x_{E} / X_{c}, h_{E} / x_{E}\right)=K_{1, R, \theta}+K_{2, R, \theta}\left(x_{E} / X_{c}\right)+K_{3, R, \theta}\left(h_{E} / x_{E}\right)
$$

The constants $K_{1-3, R, \theta}$ are provided in Table 6 .

\begin{tabular}{|c|c|c|c|}
\hline$\theta$ & $K_{1, S, \theta}$ & $K_{2, S, \theta}$ & $K_{3, S, \theta}$ \\
\hline 50 & 0.1009 & -2.5387 & 0.2173 \\
\hline 55 & 0.1327 & -2.5956 & 0.2177 \\
\hline 60 & 0.2526 & -2.5548 & 0.1655 \\
\hline 65 & 0.2764 & -2.2503 & 0.1384 \\
\hline 70 & 0.5237 & -2.2858 & 0.0505 \\
\hline 75 & -0.0209 & -1.2722 & 0.0719 \\
\hline 80 & -0.2302 & -0.5528 & 0.1721 \\
\hline 85 & -0.4057 & 0.269 & 0.1268 \\
\hline 90 & -0.2274 & 0.3979 & 0.0777 \\
\hline 95 & -0.1436 & 0.3123 & 0.1295 \\
\hline 100 & -0.478 & 0.3778 & 0.0921 \\
\hline 105 & -0.6176 & 0.7417 & 0.2848 \\
\hline 110 & -0.8433 & 0.9363 & 0.3094 \\
\hline 115 & -0.866 & 1.0756 & 0.2989 \\
\hline 120 & -0.8257 & 1.0751 & 0.5599 \\
\hline 125 & -1.0166 & 1.3315 & 0.6393 \\
\hline 130 & -1.3813 & 1.9517 & 0.9745 \\
\hline 135 & -1.4806 & 2.0694 & 1.4476 \\
\hline 140 & -1.1075 & 2.0255 & -0.2264 \\
\hline 145 & -1.3622 & 2.5277 & -0.2227 \\
\hline 150 & -1.5265 & 2.2508 & 3.2844 \\
\hline 155 & -1.5298 & 2.1625 & 4.228 \\
\hline 160 & -1.1855 & 3.0372 & -5.506 \\
\hline 165 & -1.3156 & 2.0395 & 4.454 \\
\hline
\end{tabular}

Table 6. Coefficients $K_{1-3, S, \theta}$ used by the shielding effect model. 